\title{
Re: Calcium Role in Human Carcinogenesis: A Comprehensive Analysis and Critical Review of Literature
}

\author{
Kadio $B^{1}$, Yaya $\mathrm{S}^{2}$, Basak $\mathrm{A}^{1,3}$, Djè $\mathrm{K}^{4}$, Gomes J', Mesenge $\mathrm{C}^{5}$ \\ 'University of Ottawa Faculty of Health Science, Interdisciplinary School of Health Sciences, Ottawa, Canada \\ ${ }^{2}$ University of Ottawa Faculty of Social Sciences, School of International Development and Global Studies, Ottawa, Canada \\ ${ }^{3}$ University of Ottawa, Chronic Disease Ottawa Hospital Research Institute, Ottawa, Canada \\ ${ }^{4}$ Allasane Ouattara University Faculty of Medicine, Department of Urology, Bouaké, Ivory Coast \\ ${ }^{5}$ University of Québec at Abitibi-Témiscamingue, Rouyn-Noranda, Québec, Canada
}

Cancer Metastasis Rev 2016;35:391-411. doi: 10.1007/s10555-016-9634-0.

\section{EDITORIAL COMMENT}

Calcium is the most abundant mineral in the human body especially in the bones and teeth. A few amount of total calcium is physiologically active (1\%), as signaling molecules; it plays major roles in biological systems importance of calcium has been appreciated over the years especially in cancer. Free calcium is associated to the many cellular processes such as cancer initiation, promotion, and progression. Its role is very crucial in cancer-related molecular mechanisms such as signaling pathway. In the literature, the relationship between calcium metabolism and many different cancers, such as breast, colon and prostate cancers, has been investigated. Two distinct mechanisms relating calcium homeostasis to cancer pathways have been described. The first pathway is calcium store-dependent, and the second is rather calcium influx-dependent. These mechanisms are responsible for cellular life and control mechanisms over cell death, growth, and division. As a result, calcium could also function as a first messenger in cancer. These responsible mechanisms are alterations in cell surface calcium-sensing receptor, alterations in transmembrane calcium trafficking protein expression, the role of non-membrane-bound calcium-binding proteins, resistance to apoptosis, increased compartmental calcium concentration initiating cancerous transformation, the cytoplasmic effects of high intracellular calcium, habituation of cells to lower levels of calcium. These theories may contribute to cancer research for the development of new diagnosis, prognosis, and treatment tools and options based on calcium signaling.

Fehmi Narter, MD, PhD

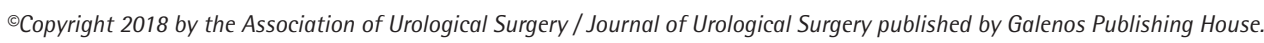

